Rev. Elev. Méd. vét. Pays trop., 1979, 32 (1) : 37-46

\title{
Effets sur Glossina palpalis gambiensis de substances médicamenteuses administrées au lapin hôte-nourricier
}

\author{
Première partie
}

\author{
par D. CUISANCE $\left({ }^{*}\right)$, E. SELLIN $\left({ }^{*}\right)$, Y. TAZE $(*)$, M. CLAIR $\left({ }^{*}\right)$, H. POLITZAR $(*)$
}

\begin{abstract}
RÉSUME
L'administration de 13 produits médicamenteux (5 antibiotiques, 1 sulfamide, 1 neuroplégique, 3 vitamines et 3 hormones) à des lapins, a provoqué, chez des femelles de G. p. gambiensis nourries sur eux, des perturbations physiologiques se traduisant, notamment, par une diminution de la longévité et de la fécondité.

Le sulfamide (Eftolon) est le plus nocif. Les hormones diminuent nettement la longévité. Le taux d'éclosion des pupes produites est inférieur à celui du lot témoin.

Les modalités d'action possibles de ces médicaments sont discutées, ainsi que leurs possibilités d'emploi chez les animaux destinés à nourrir des élevages de glossines, ou leur utilisation éventuelle dans la lutte contre ces insectes.
\end{abstract}

Les effets néfastes de modifications accidentelles ou volontaires de la composition du repas sanguin chez les glossines ont été nettement démontrés ces dernières années. De fortes perturbations de la fécondité (avortements, dégénérescences folliculaires) (14), une réduction de la longévité associée à un refus de se nourrir et de s'accoupler chez les individus jeunes (18) ont été observées chez G. morsitans orientalis et $G$. morsitans submorsitans.

JORDAN et TREWERN (6) signalent une baisse de fécondité dans une colonie de Glossina austeni nourrie sur des lapins recevant des sulfamides coccidiostatiques. NOGGE (8) souligne la baisse de longévité enregistrée chez les glossines ayant perdu leurs symbiontes intestinaux après administration de différents produits dont une tétracycline. La même observation est signalée par SOUTHWOOD et collab. (17) nourrissant $G$. morsitans sur des lapins traités à l'Ampicilline. PELL et collab. (10) montrent qu'un symbionte de type rickettsien localisé

(*) Centre I. E. M. V. T. - G. T. Z. de Recherches sur les Trypanosomoses animales B. P. 454 BoboDioulasso, République de Haute-Volta. aux ovaires est indispensable au développement normal de ceux-ci; une chute importante de la fécondité à laquelle peut s'associer une baisse de longévité est signalée par NOGGE (9) et PELL et collab. (11) à la suite de l'emploi d'un antibiotique ou d'un sulfamide supprimant les symbiontes intestinaux et ovariens de G. m. morsitans.

SCHLEIN (15) note les effets marqués de tétracyclines sur G. m. morsitans nourrie sur des lapins traités tandis que JORDAN et TREWERN (7) tentent d'analyser le mécanisme de l'action de la sulfaquinoxaline sur la fécondité de $G$. austeni et de $G$. morsitans à la suite de l'accident survenu, dans leur colonie de glossines, après modification des rations alimentaires des lapins nourriciers.

En avril 1976, époque où la colonie de G. $p$. gambiensis de Bobo-Dioulasso avait atteint l'effectif prévu de 30000 femelles (16), le passage de la saison sèche à la saison des pluies a provoqué, chez les lapins nourriciers, des pneumonies à Klebsiella pneumoniae qui ont été enrayées aux antibiotiques et aux sulfamides.

Les lapins traités ont été isolés et mis en quarantaine. Toutefois, à la suite d'erreurs de mani- 
pulations, certains de ces animaux ont été utilisés trop tôt pour nourrir la colonie de glossines, qui a alors présenté de graves désordres compromettant sérieusement sa vitalité. Après enquête, antibiotiques et sulfamides ont été fortement suspectés. Afin de confirmer cette hypothèse, une expérimentation a été entreprise faisant appel non seulement à des antibiotiques et à des sulfamides mais également à d'autres produits médicamenteux. Elle a également été effectuée dans le but de sélectionner des médicaments peu toxiques pour les glossines, ou, au contraire, de trouver des produits éventuellement utilisables dans la lutte contre ces insectes.

\section{MÉTHODOLOGIE}

L'expérimentation a porté sur des produits qui, bien que d'activité pharmaco-dynamique très différente, sont couramment utilisés en élevage. Ils ont tous été administrés, en une seule injection, à doses curatives uniquement, par voie intraveineuse, à l'exception de la Procamycine et des hormones, injectées par voie intra musculaire.

\section{Traitement des animaux}

Les produits utilisés ont été les suivants :

1) Cinq antibiotiques :

- Oxytétracycline, chlorhydrate (Terramycine, N. D.) :

$50 \mathrm{mg}(1 \mathrm{ml})$ à 1 lapin de $3,5 \mathrm{~kg}$.

- Ampicilline sodique (Totapen, N. D.) : $150 \mathrm{mg}(1,5 \mathrm{ml})$ à 1 lapin de $2,8 \mathrm{~kg}$.

- Kanamycine base, sesquisulfate associé à un sel sodique de pénicilline (Kamypen, N. D.) :

$75 \mathrm{mg}+450000 \mathrm{UI}(1 \mathrm{ml})$ à 1 lapin de $3,5 \mathrm{~kg}$.

- Spiramycine base associée à de l'acide adipique (Suanovil, N. D.) : $125 \mathrm{mg}(2,5 \mathrm{ml})$ à 1 lapin de $2,8 \mathrm{~kg}$.

- Dihydrostreptomycine, sulfate, plus pénicillate de procaïne (Procamycine, N. D.) : $250 \mathrm{mg}+250000 \mathrm{UI}(1 \mathrm{ml})$ à 1 lapin de $3 \mathrm{~kg}$.

2) Un sulfamide :

- Sulfaphénazole (Eftolon, N. D.) : $375 \mathrm{mg}(1 \mathrm{ml})$ à 1 lapin de $4,4 \mathrm{~kg}$.
3) Un neuroplégique :

-Chlorpromazine (Largactil, N. D.) : $5 \mathrm{mg}(1 \mathrm{ml})$ à 1 lapin de $2,5 \mathrm{~kg}$.

4) Trois vitamines:

- Pyridoxine, chlorhydrate (Vitamine B 6, N. D.) :

$50 \mathrm{mg}(1 \mathrm{ml})$ à 1 lapin de $2,7 \mathrm{~kg}$.

- Cyanocobalamine (Vitamine B 12, N. D.) : $250 \mu \mathrm{g}(1 \mathrm{ml})$ à 1 lapin de $3,4 \mathrm{~kg}$.

- Acide L-ascorbique (Vitascorbol N. D.) : $100 \mathrm{mg}(1 \mathrm{ml})$ à 1 lapin de $2,3 \mathrm{~kg}$.

5) Trois hormones:

- Oestradiol, benzoate (Benzogynoestryl, N. D.) :

$2 \mathrm{mg}(0,4 \mathrm{ml})$ à 1 lapin de $3 \mathrm{~kg}$.

- Oxyprogestérone, caproate (Progestérone retard, N. D.) :

$100 \mathrm{mg}(0,4 \mathrm{ml})$ à 1 lapin de $2,4 \mathrm{~kg}$.

- Testostérone, propionate (Testoviron, N. D.) :

$12,5 \mathrm{mg}(0,25 \mathrm{ml})$ à 1 lapin de $2,4 \mathrm{~kg}$.

\section{Protocole d'alimentation des glossines :}

- L'expérimentation a porté uniquement sur des glossines femelles; en effet ce sont elles qui conditionnent l'accroissement de la colonie et sa productivité, qui dépend de la fécondité des femelles et de leur longévité.

- Toutes les glossines ont été accouplées à l'âge de 3 jours avec autant de mâles âgés de 7-10 jours, puis séparées $48 \mathrm{~h}$ plus tard.

- Les glossines femelles, à jeûn, sont nourries sur le lapin $10 \mathrm{mn}$ après le traitement intraveineux ou $24 \mathrm{~h}$ après le traitement intramusculaire.

- Chaque glossine ne prend qu'un seul repas médicamenteux; seules les glossines visiblement gorgées sont retenues pour constituer un lot expérimental. Tous les repas suivants sont pris sur lapin non traité.

\section{Protocole d'expérimentation :}

- Un lot témoin de 40 femelles est nourri quotidiennement et exclusivement sur un lapin n'ayant reçu aucune injection.

- Pour chaque produit expérimenté, on a constitué trois lots différents suivant l'âge des femelles au moment de la prise du repas médicamenteux :

- 40 femelles âgées de 2 jours (avant la première ovulation) ; 


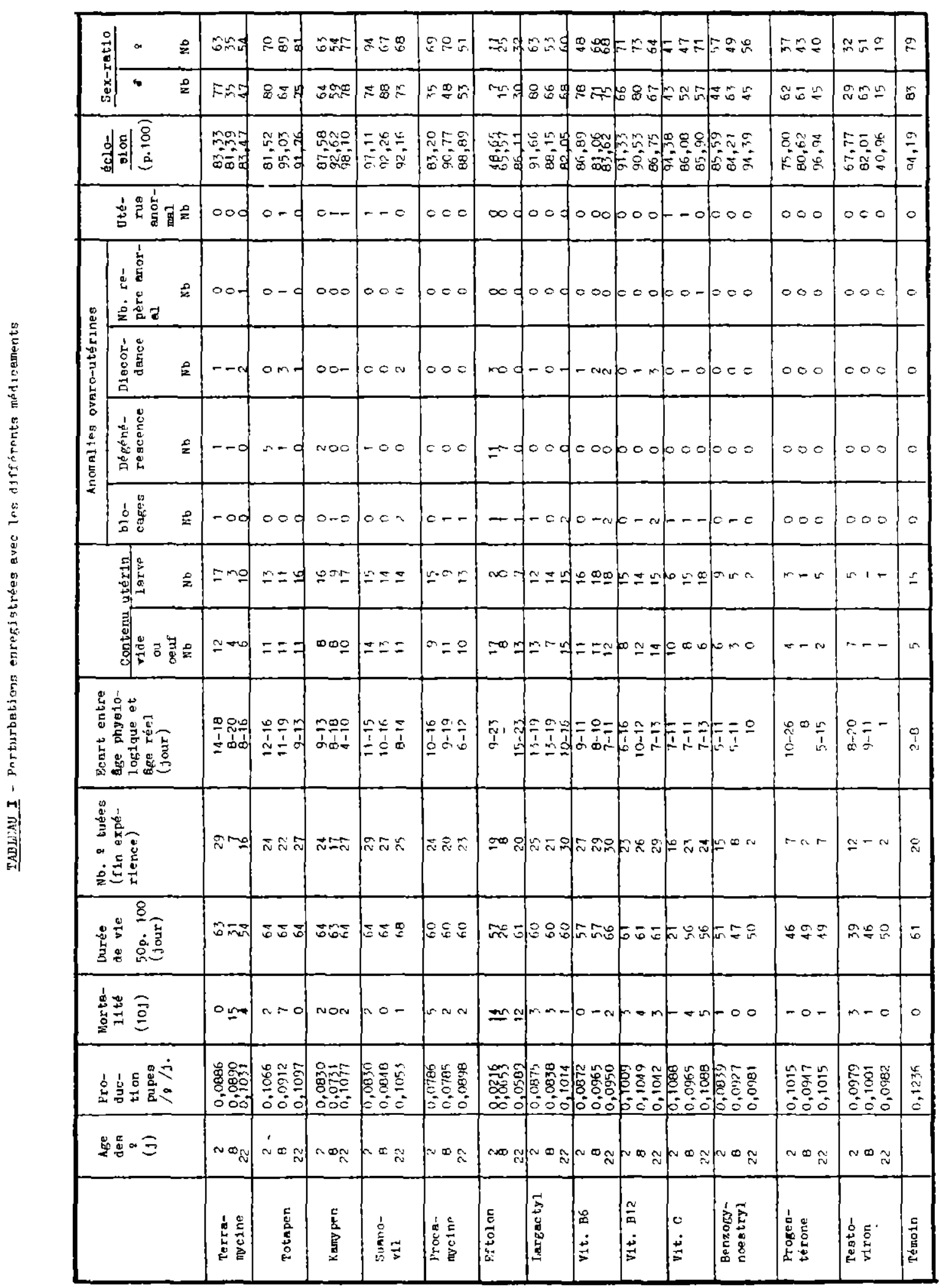


- 40 femelles âgées de 8 jours (avant la première larviposition) ;

- 40 femelles âgées de 22 jours (après la première larviposition).

La récolte quotidienne des pupes produites; le pourcentage d'éclosion et le sex-ratio des individus qui en sont issus; l'examen, après dissection, de l'appareil génital de toutes les femelles mortes en cours d'expérimentation ou sacrifiées à la fin de celle-ci ( $60^{\circ}$ jour), ont permis l'établissement des paramètres suivants : fertilité, longévité, écart entre l'âge réel et l'âge physiologique, état ovaro-utérin, état de la descendance.

\section{Fertilité}

\section{RÉSULTATS}

(Pour chaque lot, on a calculé le nombre de pupes produites et le nombre de femelles repro- ductrices par jour, à partir du $17^{\mathrm{e}}$ jour de vie qui est l'âge moyen des femelles lors de leur première larviposition.)

La fertilité est exprimée en nombre de pupes par femelle reproductrice et par jour (cf. tabl. I colonne $\mathrm{n}^{\circ} 1$ ).

Le taux de fertilité de chaque lot d'expérience, rapporté à celui du lot témoin, considéré comme égal à 100 p. 100 , est représenté sur le diagramme $\mathrm{n}^{\circ} 1$.

On constate alors que, quel que soit le produit injecté, la fertilité des femelles est inférieure à celle des témoins. Cette différence est en moyenne de 25,33 p. $100( \pm 4,20)$.

L'effet d'abaissement de la fertilité est, par ordre croissant, pour chaque produit (3 lots d'âges groupés), le suivant :

Vitamine C $(15,29$ p. 100), Vitamine B 12 $(16,40$ p. 100$)$, Totapen (17,07 p. 100), Proges-

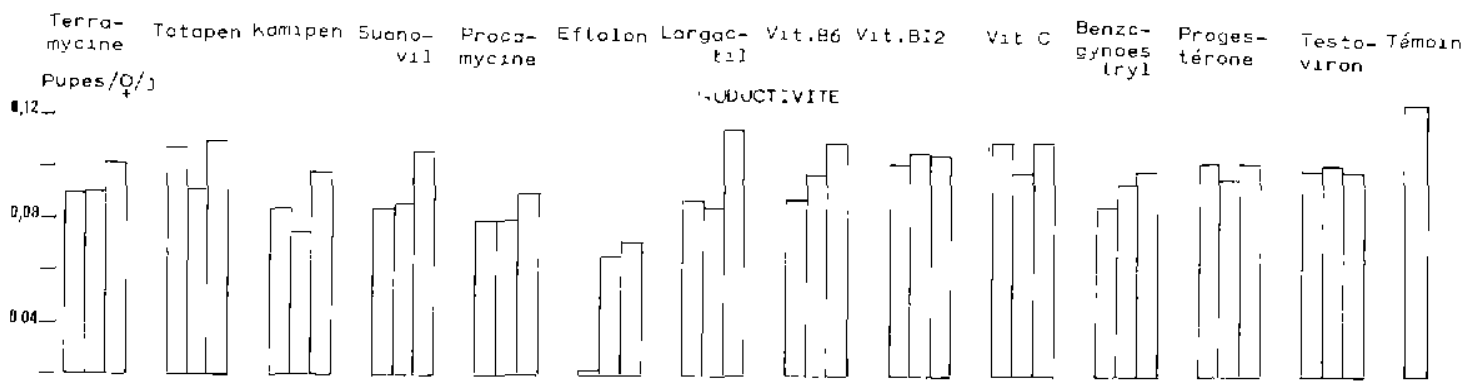

$100-$

$-$

MUE"ALITE IIU jours)

6.

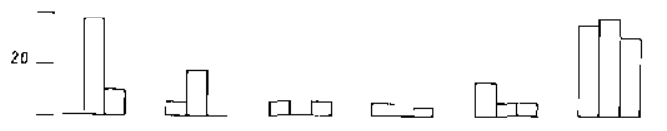

70 Jours
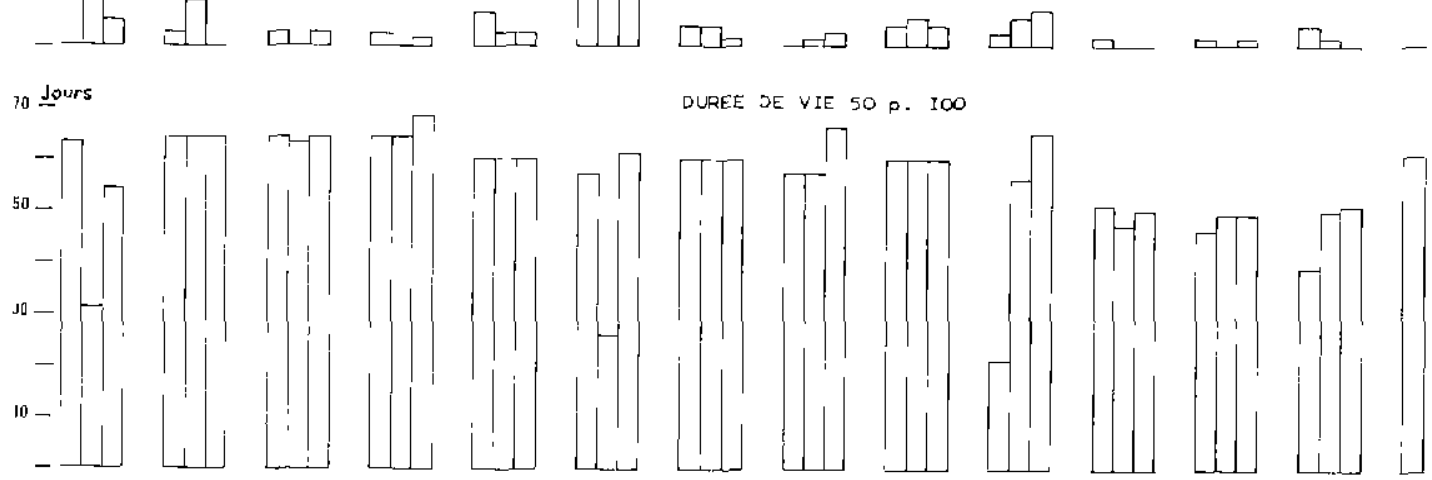

20 Jowrs
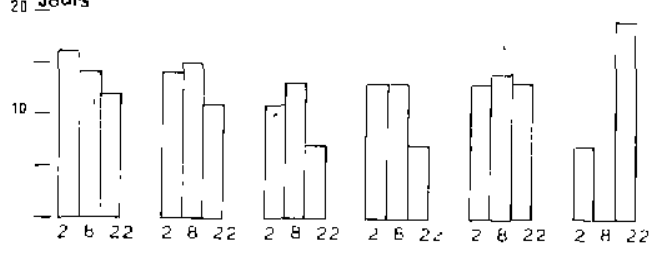

ECA IT AGE HEEL - AGE FHYSILLUGIQUE
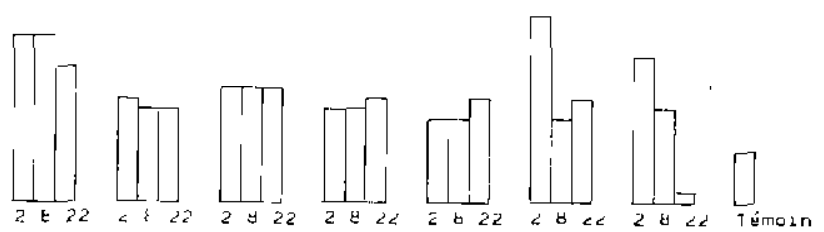
térone (19,71 p. 100), Testoviron (20,11 p. 100), Vitamine B $6(21,25$ p. 100), Terramycine (24,30 p. 100), Benzogynoestryl (25,51 p. 100), Suanovil (26,35 p. 100), Largactil (26,45 p. 100), Kamypen (28,86 p. 100$)$, Procamycine $(33,41$ p. 100), Eftolon (57,58 p. 100).

Les baisses de fertilité les plus fortes sont dues aux antibiotiques et au sulfamide. C'est avec celui-ci que l'écart est le plus important (près de 60 p. 100).

Le test $\varepsilon$ de l'écart réduit, confirme largement ces différences, très significatives par rapport au lot témoin (cf. tabl. II).

Dans la presque totalité des cas, l'effet sur la fertilité de la glossine est d'autant plus marqué que celle-ci est plus jeune. Comme précédemment, l'Eftolon est la substance dont l'action est la plus marquée : la baisse de productivité des femelles passe de 82,53 p. 100 à l'âge de 2 jours, à 47,17 p. 100 à l'âge de 8 jours et 43,04 p. 100 à l'âge de 22 jours.

\section{Longévité}

L'étude de ce paramètre repose sur deux critères : la mortalité dans les 10 jours suivant la prise du repas médicamenteux et la durée de vie 50 p. 100.

Tandis qu'aucune mortalité n'est observée dans le lot témoin dans les 10 jours suivant le repas, on relève une perte moyenne de 7,90 p. 100 sur l'ensemble des lots expérimentaux. Toutefois, la mortalité n'est significativement différente de celle du lot témoin que pour deux produits: Terramycine $\left(15,83\right.$ p. $100 ; \mathrm{X}^{2}=5,74$; d. d. $1 .=1)$ et Eftolon $\left(34,17\right.$ p. $100 ; X^{2}=18,36$; d. d. 1. = 1).

Les produits abaissant la durée de vie 50 p. 100 de façon significative sont les suivants (d. d. 1. $=78):$ Terramycine $(\mathrm{t}=3,80)$, Eftolon $(t=6,40)$, Vitamine $C(t=5,60)$ et les trois hormones, de façon accusée: Benzogynoestryl $(t=52,80)$, Progestérone $(t=71,82)$, Testoviron $(\mathrm{t}=24,44)$.

Cette action sur la longévité, très accusée dans le cas des hormones, paraît indépendante de l'âge des mouches.

\section{Anomalies ovaro-utérines}

La dissection systématique des glossines mortes et de celles sacrifiées en fin d'observation permet de mesurer les trois critères suivants :

— écart entre l'âge physiologique et l'âge réel.
- contenu utérin,

— anomalies ovaro-utérines.

\section{Ecart entre l'âge physiologique et l'âge réel}

L'appréciation de l'âge physiologique est faite selon la méthode de SAUNDERS (13) améliorée par CHALLIER (1). Il est comparé à l'âge réel des femelles, qui est connu.

D'une façon générale, il y a un décalage très net entre l'âge réel et l'âge physiologique. D'après les images ovariennes, les femelles apparaissent plus vieilles qu'elles ne le sont en réalité.

Pour tous les lots traités, cet écart est en moyenne de 11,72 jours $( \pm 3,51$ ) alors qu'il n'est que de 5 jours $( \pm 3)$ chez le témoin.

$\mathrm{Si}$ on compare chaque groupe traité (tous âges confondus) avec le témoin par un test de $t$, une différence significative apparaît très nettement (cf. tabl. Il) montrant que les femelles ayant pris un repas médicamenteux ont une avance sur leur âge réel très supérieur à celle du témoin.

L'écart le plus important se remarque dans les groupes traités avec le Largactyl (14,95 jours), la Terramycine (14,50 jours), la Procamycine (13,29 jours), la Progestérone (13,25 jours), le Totapen (13,19 jours) et l'Eftolon (13,15 jours).

Dans chaque groupe traité, l'effet du produit n'apparaît pas lié à l'âge des glossines.

Cette action marquée des produits médicamenteux sur les cycles ovariens de G. p. gambiensis est à rapprocher de la baisse de productivité des femelles, très significativement inférieure à celle du lot témoin.

Ces deux phénomènes sont liés, et sont vraisemblablement une conséquence des avortements présentés, de façon plus ou moins fréquente, par les femelles traitées.

\section{Contenu utérin}

Les dissections systématiques de l'appareil génital ont montré que, chez les témoins, $25 \mathrm{p}$. 100 des femelles ont un utérus vide ou contenant un ouf, contre 75 p. 100 ayant une larve in utero. Ces taux sont comparables à ceux trouvés par VAN DER VLOEDT et collab. (19).

Les femelles traitées s'écartent peu de ces rapports, sauf celles ayant absorbé de l'Eftolon et du Testoviron, chez lesquelles la différence est nettement significative $\left(X^{2}=19,05\right.$ et $X^{2}=4,37$; d. d. $1 .=1$ ).

Dans ces deux lots, l'inversion des rapports rappelle celles qui ont été observées par VAN 


\begin{tabular}{|c|c|c|c|c|c|c|c|c|c|c|c|c|c|}
\hline 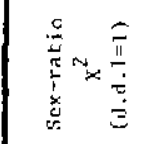 & $=1$ & 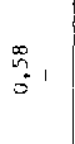 & 01 & $\begin{array}{l}\overrightarrow{0}_{0} \\
\dot{0}^{\prime}\end{array}$ & $j^{+}$ & $\vec{\Xi}$ & $\begin{array}{l}1 \\
\vdots \\
0 \\
0\end{array}$ & $\stackrel{8}{\stackrel{1}{0}}$ & $\begin{array}{l}\vec{E}_{-} \\
\vec{e}^{\prime}\end{array}$ & $\begin{array}{l}\tilde{J}_{1} \\
\dot{D}^{\prime}\end{array}$ & $\tilde{z}_{0}$ & $\dot{s}_{1}$ & 01 \\
\hline 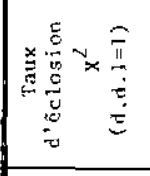 & $\vec{c}^{-}+$ & $\stackrel{g}{+}_{+}^{+}$ & $\begin{array}{l}\text { ते } \\
c^{-1}\end{array}$ & $\begin{array}{l}\overrightarrow{0} \\
0^{\prime}\end{array}$ & $\vec{m}+$ & $\begin{array}{l}\infty \\
\infty \\
\tilde{N}^{-1}\end{array}+$ & $\tilde{s}^{+}$ & $\stackrel{\infty}{\infty}_{\stackrel{\infty}{=}}^{=}+$ & $\cong 1$ & $f_{j+}$ & $\frac{R}{n^{2}}+$ & $\underset{\dot{g}}{\stackrel{8}{\sigma}}+$ & $\stackrel{\infty}{\infty}+_{\hat{\sigma}^{+}}^{+}$ \\
\hline 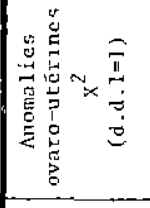 & $\begin{array}{l}\Sigma_{1} 1 \\
i_{1}\end{array}$ & $\underbrace{}_{-1}$ & $\stackrel{n}{n}{ }^{n} 1$ & $\begin{array}{l}m_{1} \\
0^{\prime}\end{array}$ & 01 & $\stackrel{\infty}{m}_{m^{?}}^{+}$ & $\stackrel{D}{p}_{?}^{\infty}$ & $\stackrel{\infty}{\varrho_{1}} 1$ & $\frac{\sigma}{0^{2}}$ & $=1$ & 0. & $=1$ & $c 1$ \\
\hline 总至 & $\stackrel{\Im}{-}+$ & $\begin{array}{l}\Xi_{1} \\
\therefore\end{array}$ & $\begin{array}{l}\text { 望, } \\
0.1\end{array}$ & $\stackrel{m}{9}$ & $\vec{i}$ & $\begin{array}{l}\text { 总+ } \\
g^{+}\end{array}$ & 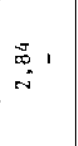 & $\stackrel{\text { s. }}{=}$ & $\begin{array}{l}\infty \\
\stackrel{\infty}{*} 1 \\
i\end{array}$ & $\because 1$ & $\begin{array}{l}\vec{E}, \\
\dot{0}\end{array}$ & $\frac{9}{2} 1$ & $\tilde{\sigma}^{+}+$ \\
\hline 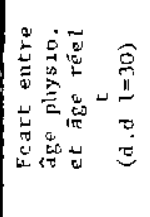 & $\frac{⿱ 5}{5}+$ & $\frac{n}{n+}+$ & $\stackrel{\infty}{\stackrel{\infty}{\infty}+}$ & $\begin{array}{l}\infty_{\infty}^{+} \\
\sigma^{+}\end{array}$ & $\begin{array}{l}\overrightarrow{1} \\
\vec{R}^{\prime}\end{array}$ & $\frac{\sigma_{n}}{\sigma^{+}}+$ & $\begin{array}{l}\frac{a}{g} \\
\frac{j}{m}+\end{array}$ & $\frac{g}{s}+$ & $\frac{8}{5}+$ & $\begin{array}{l}\vec{\infty} \\
\vec{g}^{+}\end{array}$ & $\frac{\circ}{5+}$ & $\underset{\infty}{+\infty}$ & $s^{5}+$ \\
\hline 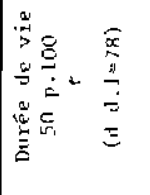 & 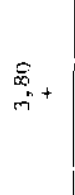 & $\begin{array}{l}8 \\
m^{2}\end{array}$ & ' & 1 & $\stackrel{8}{\&} 1$ & $q_{6}^{+}+$ & $\stackrel{\varepsilon}{\varepsilon}$ & $\stackrel{a}{a}_{-}$ & 01 & $\underset{s i}{0}+$ & $\begin{array}{l}\bar{\Xi} \\
\hat{N}^{+}\end{array}$ & $\stackrel{\tilde{x}_{\alpha}}{=}+$ & $\begin{array}{l}Z \\
\dot{\Delta}\end{array}+$ \\
\hline 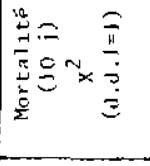 & $\vec{n}+$ & $\begin{array}{l}\infty 1 \\
0^{-1}\end{array}$ & $\begin{array}{l}m \\
0^{-1}\end{array}$ & \begin{tabular}{l}
7 \\
\multirow{0}{*}{1} \\
0
\end{tabular} & $\begin{array}{l}\infty \\
\infty \\
0 \\
0\end{array}$ & 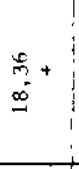 & $\underbrace{}_{-1}$ & $=1$ & 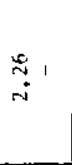 & $\begin{array}{l}\vec{F}_{1} \\
\mathrm{~N}^{\prime}\end{array}$ & $\begin{array}{l}\frac{2}{5} \\
=1\end{array}$ & $\begin{array}{l}\pi_{1} \\
0^{-}\end{array}$ & $\begin{array}{l}s_{1} \\
\vdots \\
\vdots\end{array}$ \\
\hline 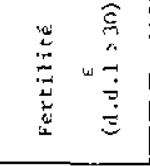 & $\begin{array}{l}8 \\
\stackrel{8}{5}+ \\
\end{array}$ & $\bar{x}^{+}$ & $\stackrel{8}{=}$ & 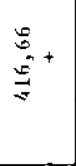 & $\begin{array}{l}8 \\
0^{-} \\
=\end{array}$ & $\begin{array}{l}\vec{m} \\
\stackrel{p}{0}^{+}\end{array}$ & $\begin{array}{l}\vec{E} \\
\stackrel{8}{\Phi} \\
\stackrel{-}{+}\end{array}$ & 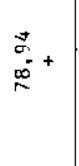 & 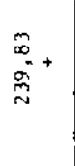 & $\begin{array}{l}\mathscr{2} \\
\stackrel{-}{\Xi}\end{array}$ & $\begin{array}{l}\frac{a}{0} \\
\vec{b}^{+} \\
\Rightarrow\end{array}$ & $\frac{0}{n^{-}}+$ & 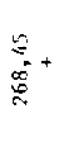 \\
\hline & 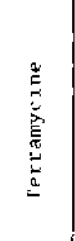 & 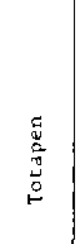 & 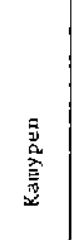 & 节 & 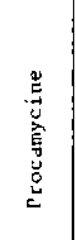 & 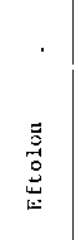 & J & 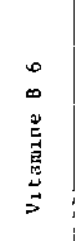 & 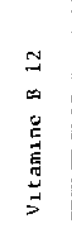 & 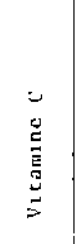 & 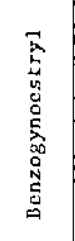 & 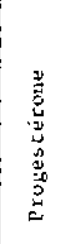 & 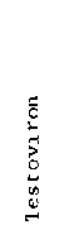 \\
\hline
\end{tabular}


DER VLOEDT (19) et nous-mêmes $(3,4)$ chez des femelles accouplées avec des mâles irradiés. La proportion de femelles ayant un utérus vide ou contenant un cuf atteint même, chez celles traitées avec l'Eftolon, 85 à 100 p. 100 .

Ces anomalies utérines, confirmées par celles qui affectent la configuration ovarienne, indiquent que : vide),

- soit l'ovulation ne s'effectue pas (utérus

- soit les cufs ovulés ne sont pas viables et sont expulsés avant d'aboutir au premier stade larvaire.

\section{Anomalies ovaro-utérines}

On a retenu cinq types d'anomalies assez facilement identifiables au moment de la dissection :
- un blocage, au niveau des ovarioles, de deux ou plusieurs aufs ;

- dégénérescence, au niveau des ovarioles ou de l'oviducte, d'un ou de plusieurs œufs;

- discordance entre la configuration ovarienne et le contenu utérin;

- " nombre repère" anormal ;

- aspect pathologique de l'utérus (mince et très allongé).

A l'exception des groupes traités aux hormones où pratiquement aucune anomalie n'est observée, on rencontre chez tous les autres des perturbations du fonctionnement ovaro-utérin. Les pourcentages (tabl. I et diagramme $n^{\circ} 2$ ) sont cependant assez faibles dans l'ensemble à l'exception de l'Eftolon qui se distingue très significativement $\left(X^{2}=13,78\right.$; d. d. $\left.1 .=1\right)$ du groupe

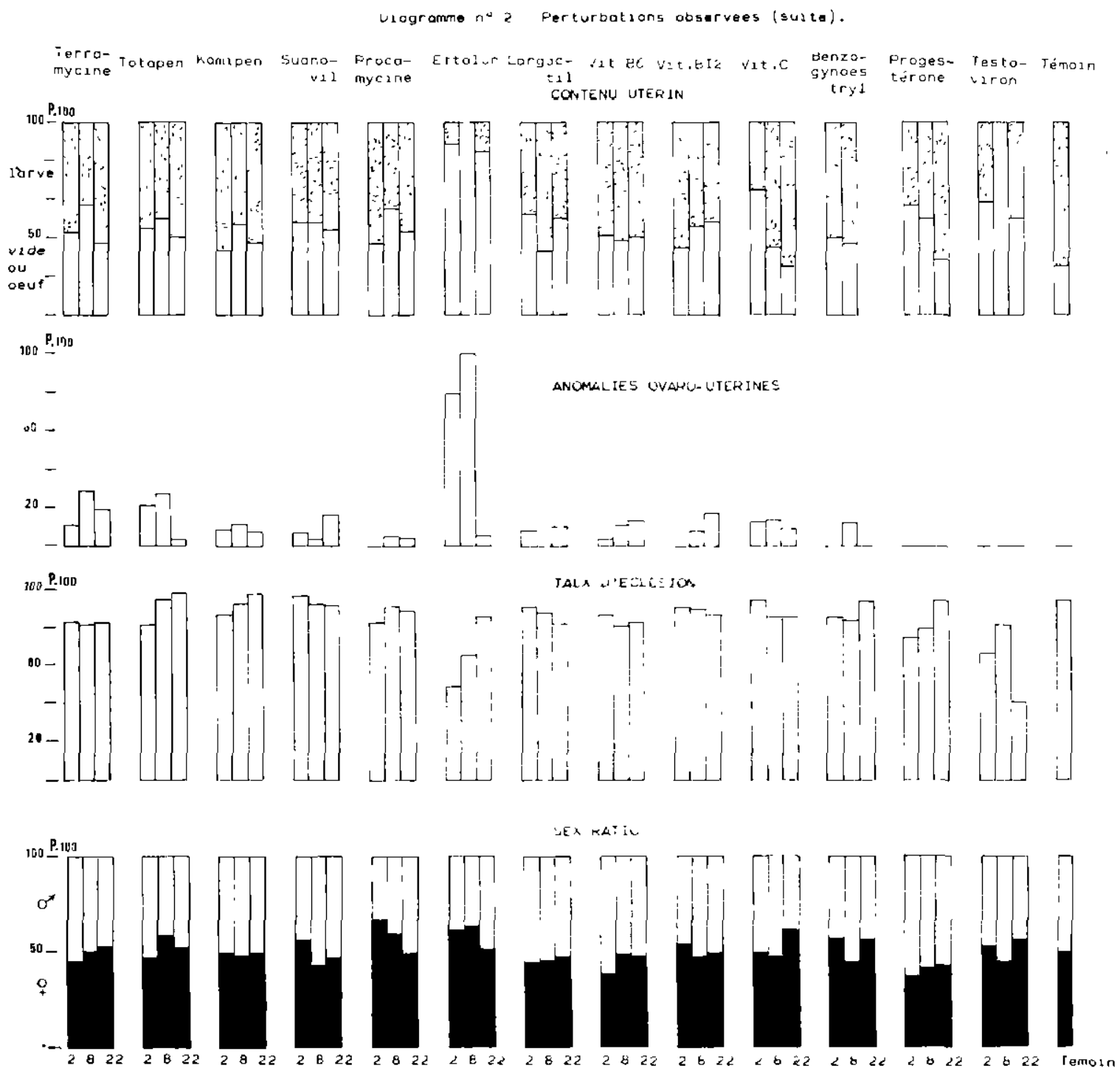


témoin avec deux lots (2 jours et 8 jours) présentant 79 p. 100 et 100 p. 100 d'images ovaroutérines pathologiques.

Terramycine et Totapen semblent, après l'Eftolon, les plus actifs mais sans atteindre la significativité $\left(\mathrm{X}^{2}=2,06\right.$ et $\left.\mathrm{X}^{2}=2,46\right)$. On remarque que les blocages et les discordances sont présents chez les groupes traités avec les antibiotiques, le sulfamide, le neuroplégique et les vitamines. Pour le groupe traité aux antibiotiques, on observe en outre des images de dégénérescence d'oufs. Les discordances confirment encore l'existence d'avortements.

\section{DESCENDANCE}

Deux paramètres ont semblé intéressants : le taux d'éclosion des pupes produites par les femelles traitées et le sex-ratio des imagos qui en sont issus.

\section{Taux d'éclosion}

L'impact des produits médicamenteux sur le taux d'éclosion des pupes est général, mais assez limité (taux inférieur de 8 p. 100 à celui des témoins). Il est cependant très marqué avec le Testoviron $\left(X^{2}=45,81 ;\right.$ d. d. $\left.1 .=1\right)$ et avec l'Eftolon $\left(X^{2}=32,88\right.$; d. d. $\left.1 .=1\right)$, sensible avec la Progestérone $\left(X^{2}=13\right.$; d. d. I. $=1)$, la Terramycine $\left(X^{2}=12,35\right.$; d.d. $1 .=1)$, la Vitamine B $6\left(X^{2}=11,86\right.$; d. d. $1 .=1)$ et peu important avec les autres produits (cf. tabl. II et diagramme $\mathrm{n}^{\circ} 2$ ).

\section{Sex-ratio}

Le rapport des sexes à l'éclosion n'est pas affecté par les traitements.

Seule la Procamycine paraît avoir une légère influence, en faveur des femelles, avec un $\mathrm{X}^{2}$ à peine significatif au risque de 5 p. 100 .

\section{DISCUSSION}

Les effets observés sont très variables, mais ils affectent en priorité la fertilité puis la longévité et, dans une moindre mesure, la descendance.

L'intensité de l'effet est manifestement liée au produit utilisé. En groupant le nombre de paramètres étudiés, pour chaque produit, on obtient la classification suivante, par effet décroissant :
- Eftolon : 7 ;

- Terramycine, Testoviron: 5;

- Procamycine, Vitamine C, Benzogynoestryl, Progestérone : 4 ;

- Totapen, Largactil, Vitamine B 6: 3; - Kamypen, Suanovil, Vitamine B 12: 2.

Quel est donc le mode d'action de chacun d'eux?

SOUTHWOOD (17) a montré que les antibiotiques (ampicilline) affectaient le processus de digestion du sang.

PELL (10) et NOGGE (9) ont souligné l'importance des symbiontes, tant ovariens qu'intestinaux, dans la physiologie de la reproduction de la glossine (synthèse des vitamines du groupe B).

Les bactéries de l'intestin sont la principale source d'acide folique (acide ptéroyl-glutamique) chez les mammifères. Cette synthèse est inhibée par les sulfamides.

P. E. PELL (11) signale le même phénomène chez les glossines (G. austeni), où l'acide folique est utilisé, au cours de la synthèse de l'ADN, dans les cellules nourricières des follicules ovariens. La disparition des symbiontes entraîne des dégénérescences folliculaires, phénomène précisément constaté ici avec les antibiotiques et l'Eftolon.

Le complexe Terramycine et Auréomycine utilisé par voie intra-péritonéale ou par voie orale chez le lapin nourricier provoque, après un seul repas, la destruction du mycétome d'où une mortalité importante des glossines femelles et une baisse de vitalité des descendants ( $\mathrm{SCH}$ LEIN, (15)). Cet auteur propose même d'en faire une méthode de lutte contre les glossines par traitement des animaux domestiques, hôtes nourriciers des glossines dans la nature.

JORDAN et TREWERN (7), 'associant sulfaquinoxaline et pyriméthamine, constatent que seule la première est très active sur la fécondité ; elle réduit le nombre de pupes produites et provoque des avortements après absorption continue à petites doses. Elle réduit également le taux d'éclosion des pupes produites. Elle agirait en interférant avec le métabolisme du folate au niveau des ovaires.

Il apparaît donc que les antibactériens majeurs (antibiotiques et sulfamides) agissent sur les glossines en détruisant leurs symbiontes, intestinaux ou ovariens, d'où carence en vitamines du groupe $B$, en particulier en acide folique qui intervient dans le métabolisme des cellules germinales. 
Les perturbations enregistrées chez les glossines utilisées dans la présente étude se rapprochent très fortement des conclusions des auteurs cités plus haut. Toutefois, on remarque que, parmi les antibactériens, l'activité est variable suivant le produit utilisé et que l'Eftolon (sulfamide) se distingue nettement par l'intensité de ses effets. De plus, neuroplégique, vitamines et hormones sont également nocifs, ce qui implique d'autres mécanismes d'action que ceux énoncés par les différents expérimentateurs. On note en particulier l'effet des hormones sur la longévité des femelles. SCHLEIN (15) suspecte un effet proprement toxique, ce qui est vraisemblable.

L'activité, chez la glossine, des produits utilisés, est également fonction de la dose employée. PELL et collab. (11) constatent sur G. m. morsitans un effet net à 300 p. p. m. de sulfaquinoxaline dans la ration du lapin nourricier et pas d'effet à 75 p. p. m.

A doses thérapeutiques normales, les médicaments sont tous actifs sur les glossines, qu'ils soient utilisés à dose unique (un seul repas médicamenteux) donc sur un mode curatif, ou à doses répétées (repas multiples) donc sur un mode prophylactique. Les doses fortes agiraient sur la longévité, les doses faibles sur la fécondité (JORDAN et TREWERN, 7).

Ces auteurs utilisaient la sulfaquinoxaline pour prévenir la coccidiose du lapin; dans notre élevage nous traitions curativement des lapins atteints de pneumonies. Dans aucun cas, les doses n'étaient excessivement élevées, ce qui explique que la fertilité ait été plus affectée que la longévité.

L'activité des médicaments est certainement fonction de l'âge de la glossine au moment de la prise du repas. SCHLEIN (15) signale une sensibilité décroissante de G.m. morsitans avec l'âge. Dans notre observation, cette tendance n'est nette qu'en ce qui concerne la production de pupes.

Signalons enfin que, d'après PELL et SOUTHERN (11) et JORDAN et TREWERN (7), l'effet nocif est fonction de l'espèce de glossine : $G$. austeni est plus sensible que $G, m$. morsitans à la sulfaquinoxaline, mais l'influence du sexe n’a pas été étudiée.

\section{CONCLUSION}

Cette expérimentation visait à étendre la gamme des produits déjà expérimentés par certains auteurs (sulfaquinoxaline, tétracyclines, ampicilline). Les médicaments que nous avons testés ont été choisis parmi ceux qui sont assez couramment utilisés dans l'élevage des animaux nourriciers, le lapin notamment.

Antibiotiques, sulfamide, neuroplégique, vitamines et hormones provoquent tous, après une seule absorption, des perturbations sensibles sur la biologie de G. p. gambiensis; la plus grave est une atteinte du potentiel reproducteur. L'action des sulfamides et des antibiotiques, démontrée par les précédents auteurs, se confirme jci et l'Eftolon (sulfaphénazole), se révèle un médicament d'une haute nocivité pour la glossine.

Le mécanisme d'action est vraisemblablement un effet destructeur des symbiontes intestinaux et ovariens indispensables à certaines synthèses vitaminiques. Ils agissent principalement sur la fertilité des femelles à la manière d'agents stérilisants.

Toutefois, le neuroplégique utilisé, les vitamines et les hormones sont également efficaces et leur mode d'action ne peut être rattaché au précédent. Ils agissent à un moindre degré sur la fertilité. En ce qui concerne la vitamine $\mathrm{C}$ et les hormones, leur action nette sur la longévité fait penser à un effet toxique.

Ces résultats permettent d'expliquer l'accident survenu dans la colonie de $G$.p. gambiensis en avril 1976, époque où des lapins traités à la procamycine et aux tétracyclines ont été remis trop tôt en service. On a alors noté une forte mortalité tandis que la production de pupes a été réduite de 54 p. 100 en l'espace d'un mois nécessitant l'élimination de 9400 femelles devenues improductives.

Si tous les produits utilisés ont eu un effet néfaste, il n'est pas interdit de penser que d'autres pourraient avoir un effet bénéfique. Une nouvelle série de substances médicamenteuses sera prochainement expérimentée.

11 faut noter enfin que des modifications sanguines, non liées à l'administration de substances médicamenteuses, mais dues à des processus pathologiques (anémie) ou immunologiques (anticorps anti-salive de glossines), sont susceptibles de porter atteinte aux glossines. 


\section{SUMMARY}

\section{Effect of drugs given to the feeding host (rabbit) on Glossina palpalis gambiensis.}

Rabbits were given thirteen drugs (5 antıbiotics, /sulfamide, /neuroleptic, 3 vitamins and 3 hormones).

Physiological disturbances, especially diminution of longevity and fertility, were observed in G. p. gambiensis fed on these animals.

Sulfamide (Eftolon) is the most noxious. Hormones shorten fly longevity and the eclosion rates of pupa are inferior to those of the control batch.

The possible conditions of application of these drugs are discussed as well as their administration to feeding-hosts in glossina rearing centres or their prospective use for the insect control.

\section{RESUMEN}

Efectos sobre Glossina palpalis gambiensis de medicamentos administrados al conejo huesped-nutricio.

La administración de trece productos medicamentosos (5 antibióticos, 1 sulfamida, 1 neuropléjico, 3 vitaminas y 3 hormonas) a conejos provocó, en las hembras de G.p. gambiensis alimentadas sobre ellos, perturbaciones fisiologicas manifestandose, principalmente, por una disminución de la longevidad y de la fecundidad. La sulfamida (Eftolon) es la más nociva. Las hormonas disminuyen mucho la longevidad. La tasa de nacimiento de las pupas producidas es inferior a la del grupo testigo.

Se discuten las modalidades de acción posibles de dichos medicamentos, asi como sus posibilidades de empleo en los animales destinados a alimentar las crias de glosinas, o su utilización eventual para la lucha contra estos insectos.

\section{BIBLIOGRAPHIE}

1. CHALliER (A.). Amélioration de la méthode de détermination de l'âge physiologique des glossines. Etudes faites sur Glossina palpalis gambiensis. Vanderplank 1949. Bull. Soc. Path. exot., 1965, 58 (2) : 250259.

2. HILL (P.), SAUNDERS (D. S.), ALLAN CAMPBELL (J.). The production of «symbiont-free » Glossina morsitans and an associated loss of female fertility. Trans. r. Soc. trop. Med. Hyg., 1973, 67 (5) : 727-728.

3. I. E. M. V. T. Rapport annuel 1976, Centre de Recherche sur les Trypanosomiases Animales, BoboDioulasso (Haute-Volta), $82 \mathrm{p}$.

4. I. E. M. V. T. Rapport annuel 1977, Centre de Recherche sur les Trypanosomiases Animales,BoboDioulasso (Haute-Volta), $90 \mathrm{p}$.

5. ITARD (J.). Cycle de l'oogenèse chez les femelles de Glossina tachinoides West. et détermination de l'âge physiologique. Rev. Elev. Méd. vét. Pays trop., 1966, 19 (3) : $331-350$.

6. JORDAN (A. M.), TREWERN (M. A.). Sub-lethal effect of sulfaquinoxaline on the tsetse fly, Glossina austeni Newst. Nature, London, 1973, 245: 462.

7. JORDAN (A. M.). TREWERN (M. A.). Sulfaquinoxaline in host diet as the cause of reproductive abnormalities in the tsetse fly (Glossina). Ent. exp. appl., 1976, $19: 115-129$.

8. NOGGE (C.). Investigations on the role of symbiontes in tsetse flies (Glossina morsitans). Proc. 3rd int. Congr. Parasit., Munich, 1974.

9. NOGGE (C.). Sterility in tsetse fies (Glossina morsitans Westwood) caused by loss of symbionts. Experientia, 1976, $32: 995$.

10. PELL (P. E.), SOUTHERN (D. I.). Symbionts in the female tsetse fly Glossina morsitans morsitans. Experientia, $1975, \mathbf{3 1}$ : 650-651.

11. PELL (P. E.), SOUTHERN (D. I.). Effect of the coccidiostat, sulfaquinoxaline, on symbiosis in the tsetse fly, Glossina species. Microbios letters, 1976, 2 : 203-211.
12. SAUNDERS (D. S.). Determination of physiological age for female Glossina morsitans. Nature, London, 1960, 186 : 651.

13. SAUNDERS (D. S.). The ovulation cycle in Glossina morsitans West. (Diptera-Muscidae) and a possible method of age determination for female tsetse flies by the examination of their ovaries. Trans. r. ent. Soc. London, 1960, 112 (9) : 221-238.

14. SAUNDERS (D. S.). Reproductive abnormalıties in the tsetse fly, Glossina morsitans orientalis Vanderplank, caused by a maternally acting toxicant in rabbit food. Bull. ent. Res, 1971, $60: 431-438$.

15. SCIILEIN (Y.). Lethal effect of tetracycline on tsetse flies following damage to bacterioid symbionts. Experientia, $1977,33: 450$.

16. SELLIN (R.), POLITZAR (H.), CUISANCE (D.), CLAIR (M.). L'élevage de Glossina palpalis gambiensis Vanderplank 1949 (Diptera, Muscidae) à Bobo-Dioulasso (Haute-Volta). Rev. Elev. Méd. vét. Pays trop., 1977, 30 (1) : 41-49.

17. SOUTHWOOD (T. R. E.), KHALAF (S.), SINDEN (R. E.). The microorganisms of tsetse flies. Acta trop., 1975, 32: 259-266.

18. TURNER (D. A.), MARASHI (M. H.). A second incident of reproductive abnormalities in colonized Glossina morsitans morsitans Westw., caused by a maternally acting toxicant in rabbit food. Trans. $r$. Soc. trop. Med. Hyg., 1973, 67 : 292-293.

19. VAN DER VLOEDT (A. M, V.), TAHER (M.), CZOCK (K.H.), MALEKGHASSEMI (B.), HASELBERGER (N.). Laboratory studies on the sexual sterilization of the Tsetse fly Glossina palpalis palpalis (Robineau-Desvoidy) by ionizing radiation. II. Ovarian configuration and uterine content of females mated by irradiated males. Joint F. A. O./I. A. E. A. Research Coordination Meeting on the Sterile Insect Technique for Tsetse Fly Eradication or Control, 13-20 novembre 1976, Bobo-Dioulasso (Haute-Volta). $11 \mathrm{p}$. 